This is an electronic reprint of the original article. This reprint may differ from the original in pagination and typographic detail.

Author(s): Rantakokko, Merja; Portegijs, Erja; Viljanen, Anne; Iwarsson, Susanne; Kauppinen, Markku; Rantanen, Taina

Title: $\quad$ Changes in life-space mobility and quality of life among community-dwelling older people: a 2-year follow-up study

Year: $\quad 2016$

Version:

Please cite the original version:

Rantakokko, M., Portegijs, E., Viljanen, A., Iwarsson, S., Kauppinen, M., \& Rantanen, T. (2016). Changes in life-space mobility and quality of life among communitydwelling older people: a 2-year follow-up study. Quality of Life Research, 25(5), 11891197. https://doi.org/10.1007/s11136-015-1137-x

All material supplied via JYX is protected by copyright and other intellectual property rights, and duplication or sale of all or part of any of the repository collections is not permitted, except that material may be duplicated by you for your research use or educational purposes in electronic or print form. You must obtain permission for any other use. Electronic or print copies may not be offered, whether for sale or otherwise to anyone who is not an authorised user. 


\section{Changes in life-space mobility and quality of life among community-dwelling older people: A two-year follow-up study.}

Merja Rantakokko, $\mathrm{PhD},{ }^{1}$ Erja Portegijs, $\mathrm{PhD},{ }^{1}$ Anne Viljanen, $\mathrm{PhD},{ }^{1}$ Susanne Iwarsson, $\mathrm{PhD},{ }^{2}$ Markku Kauppinen, MSc, ${ }^{1}$ Taina Rantanen, $\mathrm{PhD}^{1}$

${ }^{1}$ University of Jyvaskyla, Gerontology Research Center and Department of Health Sciences, Jyväskylä, Finland

${ }^{2}$ Department of Health Sciences, Lund University, Sweden

Corresponding author:

Merja Rantakokko

Gerontology Research Center and Department of Health Sciences, P.O. Box 35, FI-40014 University of Jyväskylä, Finland

E-mail: Merja.rantakokko@jyu.fi

tel. +358408053589

Erja Portegijs, $\mathrm{PhD}$,

Anne Viljanen, $\mathrm{PhD}$,

Markku Kauppinen, MSc

Taina Rantanen, $\mathrm{PhD}$

Gerontology Research Center and Department of Health Sciences, P.O. Box 35, FI-40014 University of Jyväskylä, Finland

Susanne Iwarsson, $\mathrm{PhD}$

Department of Health Sciences, Lund University

Box 117, 22100 Lund, Sweden

Word count: 3281

Running head: Changes in life-space and quality of life 


\begin{abstract}
PURPOSE: Life-space mobility refers to the spatial area in which a person moves in daily life, taking into account distance, frequency and assistance needed. The aim was to examine how changes in life-space mobility are associated with changes in quality of life (QOL) over a two-year period.
\end{abstract}

METHODS: Community-dwelling people aged 75-90 years $(n=848)$ were interviewed faceto-face in their homes and followed up annually for two years. QOL was assessed with the short version of the World Health Organization QOL assessment short version (WHOQOLBREF) (range 0-130, higher scores indicate better QOL). Life-space mobility was assessed with the Life-Space Assessment (range 0-120, higher scores indicate better life-space mobility). Lower extremity performance was objectively measured with the Short Physical Performance Battery (SPPB). Cognitive impairment was assessed using the Mini-Mental State Examination. Chronic conditions and years of education were self-reported. Data were analysed with Generalized Estimation Equation models.

RESULTS: The mean life-space score at baseline was 63.9 \pm SD 20.6 and mean QOL score $100.3 \pm 11.8$. Over the follow-up, the QOL score decreased to $95.0 \pm 13.8$ across the total study sample. The decrease in QOL score was somewhat higher among those whose life-space mobility score declined $>10$ points during the follow-up compared to those whose life-space remained stable or improved, even after adjustment for age, gender, number of chronic conditions, cognitive impairment, SPPB and education.

CONCLUSIONS: Decline in life-space mobility is associated with decline in QOL. The results highlight the importance of ensuring continuous possibilities for out-of-home mobility in maintaining QOL among older people.

KEY WORDS: Participation, Aging, Outdoor activity, Life-space, Wellbeing 


\section{INTRODUCTION}

Maintaining quality of life (QOL) as people age is an important public health goal. QOL is a multidimensional concept which reflects the total wellbeing of a person and has been defined as "individuals' perception of their position in life in the context of the culture and value systems in which they live and in relation to their goals, expectations, standards and concerns" [1]. Known correlates of QOL in old age include, for example, cognitive functioning, health and functional abilities, and socioeconomic situation [2-5]. Furthermore, satisfaction with possibilities for participation in life situations [6] is one of the key dimensions of QOL in old age [7]. Possibilities for participation are threatened in old age by progressive loss of physical and psychological capacity, particularly if supportive interventions are inadequate [8].

The most common restrictions on participation of older people involve mobility outside the home [9]. Going outdoors is essential for the ability to continue valued activities outside the home, as well as for a sense of freedom and independence in old age [10]. It has been shown that participation restriction, whether it concerns physical activity [11] or social activities [12, 13], and exposes older people to poor QOL. However, longitudinal knowledge is lacking on how changes in opportunities for participation in different life situations outside the home affect QOL among older people.

In the present study, life-space mobility [14] is examined as an indicator of participation. Life-space mobility refers to the spatial area (bedroom, home, outside home, neighbourhood, town, distant locations) in which a person moves in daily life, taking into account distance, 
frequency and assistance needed [14]. By taking into account not only the frequency of movement outdoors, but also the need for assistance and the extent of the physical environment in which the individual moves, the life-space mobility offers a way to evaluate the individual's actual mobility in daily life and access to various community amenities [15], and thus also describes the opportunities for participation in different life events outside the home. Life-space mobility correlates with lower extremity performance [16], but is not totally dependent on the ability to walk, climb stairs or maintain balance, as other modes of transportation besides walking also contribute to life-space mobility. Decline in life-space mobility is known to lead to adverse health outcomes such as falls and fractures [17], development of frailty [18], institutionalization [19], and even mortality [20, 21]. We have previously reported that larger life-space and good QOL coincide among older people, regardless of their health status [22]. However, since individuals' life-space, as well as QOL, may fluctuate over time [15], longitudinal studies are needed to provide knowledge on such dynamics.

Accordingly, the aim of this study was to examine how changes in life-space mobility are associated with changes in QOL over a two-year period among 75- to 90-year-old community-dwelling people. 


\section{METHODS}

\section{Study design and participants}

This study forms a part of the "Life-space mobility in old age" (LISPE) project, which is a two-year prospective cohort study on individual and environmental determinants of life-space mobility and QOL among community-dwelling older people in central Finland. The study design, methods and non-response analyses have been reported in detail previously [23]. A random sample of 2550 community-dwelling 75 - to 90 -year-old residents of the municipalities of Jyväskylä and Muurame was drawn from the national population register. They were contacted by letter and over the phone to enquire about their willingness and to assess their suitability to take part in the study. The inclusion criteria were: communitydwelling in the study area, and able to communicate. A total of 848 eligible people agreed to participate and were interviewed in their homes during spring 2012. Of these, 816

participated in the one-year follow-up and 761 participated in the two-year follow-up. During the two-year period, 41 participants died, 15 were admitted to institutional care, and 12 were not re-interviewed due to decline in the ability to communicate. Other reasons for attrition were moving outside the study area $(n=6)$, poor health $(n=5)$, not willing to continue $(n=6)$, and not reached $(\mathrm{n}=2)$. The study flow is shown in Figure 1 .

\section{The one- and two-year follow-ups}

Prior to the follow-up data collection, updated information on vital status, addresses, telephone numbers, and date of relocation was gathered from population registers. If a person had been admitted to long-term institutional care or was no longer a resident in the study area, that person was not contacted again. 
The first follow-up (FU1) was conducted by means of telephone interviews one year (mean $362 \pm 9.3$ days) after the baseline assessment. The second follow-up (FU2) was conducted by means of postal questionnaires and telephone interviews two years (mean $721 \pm 8.0$ days) after the baseline assessment. The questionnaire was mailed to the participants 2-3 weeks prior to the telephone interview and comprised items on QOL, hearing, mood, and barriers to outdoor mobility. Upon return, the questionnaire was checked and missing information added during the telephone interview, which covered topics related to life-space mobility, health, functioning, autonomy, physical activity and changes in the life situation of the participants. Participants who could not be interviewed over the telephone due to hearing problems were offered a possibility to take part in a face-to-face home interview in their homes (FU1 n=2, FU2 n=3) or to answer all the study questions via a postal questionnaire (FU1 n=14, FU2 $\mathrm{n}=10)$.

\section{Measurements}

\section{Quality of Life}

QOL was assessed with the short version of the World Health Organization QOL WHOQOLBREF) [1] scale in a face-to-face interview at baseline and via a postal questionnaire at the two-year follow-up. The WHOQOL-BREF captures individuals' perceptions in the context of their culture and value systems, and their personal goals, standards and concerns. The 26-item scale comprises four domains; physical health (7 items), psychological health (6 items), social relationships (3 items), and environment (8 items), and also includes one item on general health and one on overall QOL. A total QOL score for all the domains combined was calculated. The total score ranges from 0 to 130, higher scores indicating better QOL [1]. 


\section{Life-space mobility}

Life-space mobility was assessed using the University of Alabama at Birmingham (UAB) Study of Aging Life-Space Assessment (LSA) [14] in a face-to-face interview at baseline and in the telephone interviews at the first and second follow-ups. The LSA captures mobility based on the distance through which a person reports moving during the 4 weeks preceding the assessment. The items establish movement patterns on specific life-space area, ranging from movement within one's dwelling to movement beyond one's town. For each life-space area (bedroom, home, outside home, neighbourhood, town, beyond town), participants were asked to state how many days a week (daily, 4-6 times a week, 1-3 times a week, less than once a week) they attained that area and whether they needed help from another person or from assistive devices. The calculated life-space mobility score reflects distance, frequency and independence of movement. Scoring ranges from 0 to 120 , with higher scores indicating better life-space mobility. The test-retest reliability of the Finnish version of the LSA has been found acceptable (intra-class correlation coefficient $=0.72)[24]$.

\section{Covariates}

Age and gender were derived from the national registers. Other covariate information was obtained in the face-to-face interview at baseline. Self-reported years of education were used as a socioeconomic indicator [25]. The number of self-reported physician-diagnosed chronic conditions was calculated on the basis of responses to a list of 22 diseases and an additional open-ended question asking about any other physician-diagnosed chronic conditions [16]. The relevance of the diseases reported in the open question was confirmed by a physician. Cognitive impairment was assessed with the Mini-Mental State Examination (MMSE) [26]. 
Lower extremity performance was objectively assessed by the Short Physical Performance Battery (SPPB)[27], which included assessments of standing balance, walking speed over 2.44 meters, and timed chair rises (five times). Each task was rated from 0 to 4 points and a sum score calculated (range 0-12) when at least two tests were completed. Nine participants had missing information on lower extremity performance and were excluded from the analysis. Higher scores indicate better lower extremity performance.

\section{Statistical analyses}

Characteristics of the participants were described using means and standard deviations (SD), or percentages in accordance with the categorization based on changes in life-space mobility. Differences between categories were tested with the Chi Square test or one-way analyses of variance (ANOVA). The QOL and life-space mobility scores were normally distributed. To calculate the change in the QOL score and life-space mobility score over the two-year followup, paired samples t-tests was used.

A decline in the life-space mobility score of $>10$ has been determined as a clinically meaningful change $[14,24,28]$ and was used as a cut-off for life-space mobility decline in the present study. Based on the changes in their life-space mobility over the follow-up, people were categorized as follows: 'Maintained', the life-space mobility score remained stable or improved slightly over both follow-ups; 'Late decline', the life-space mobility score remained stable at the first follow-up and decreased by more than 10 points at the second follow-up; 'Early decline', the life-space mobility score decreased $>10$ points at the first follow-up, but then remained stable at the second follow-up; and 'Constant decline', the life-space mobility score decreased $>10$ points at the first and second follow-ups. A lifespace mobility score was available for 806 participants at the 1-year follow-up and for 757 
participants at the 2-year follow-up. Sufficient information on life-space mobility at both measurement points was available for a total of 751 participants, who were then included in the analyses.

The overall QOL index was calculated for those who had answered to at least $80 \%$ of the items in the QOL questionnaire [1]. For three participants, baseline information in five or more QOL items was missing, and hence for these cases a QOL score was not calculated and they were not included in the analyses. At the two-year follow-up, information on QOL was available for 706 participants.

The association between changes in QOL and changes in life-space mobility was studied by constructing Generalized Estimation Equations (GEE) models [29] with an unstructured working correlation matrix using change in QOL as the outcome. In case of a missing data on the outcome variable (change in QOL), multivariate imputation by the chained equations (MICE) procedure [30] in SPSS /GEE (version 20.0) was used in the GEE modelling. Data on QOL were imputed for 45 people, and thus the analyses were based on data from 751 participants. The sensitivity analyses indicated no material differences in the associations due to imputation. Those who died $(n=41)$ or were admitted to institutional care $(n=15)$ during the follow-up were not included in the analyses.

First, the GEE model was adjusted for gender and age, and then the covariates (cognitive functioning, number of chronic conditions, physical performance and years of education) were added to the model one by one until the final model included all the covariates. The group difference represents the level difference in the QOL score and group by time interaction term tested represents the difference in time-related change in the QOL score between the different life-space mobility categories. 
Since no gender by life-space mobility interaction was observed for QOL $(\mathrm{p}=.170)$, men and women were included in the same models and the models were adjusted for gender. A value of $p<.05$ was taken as the level of statistical significance. Analyses were performed using IBM SPSS version 22.0 (SPSS Inc., Chicago, IL).

\section{RESULTS}

The mean age of the participants at baseline was $80.6(\mathrm{SD}=4.3)$, and $62 \%$ of them were women. The mean QOL score was $100.3(\mathrm{SD}=11.8)$ at baseline and $95.0(\mathrm{SD}=13.8)$ at the two-year follow-up.

\section{Changes in life-space mobility}

The mean life-space score at baseline was $63.9(\mathrm{SD}=20.6)$. Table 1 shows changes in the lifespace mobility scores at each assessment point over the two-year follow-up period according to the categorization based on changes in life-space mobility. Over the two years, the mean change in the life-space mobility score was -3.8 points (SD 16.6, $\mathrm{p}<.001$ ), ranging from -82 to +54 points.

The baseline characteristics of the participants according to the categorization based on changes in life-space mobility are shown in Table 2 and the mean values of QOL at baseline and at the two-year follow-up point are shown in Table 3. Those with late decline in lifespace mobility had statistically significantly lower MMSE scores at baseline compared to those in the other categories. No differences were found in age, gender, number of chronic conditions, years of education, lower extremity performance or the baseline QOL score $(p=.236)$ between the participants in the different life-space mobility categories. 
Changes in QOL according to changes in life-space mobility

Across the study population, the mean change in the QOL score was -6.3 points $(\mathrm{SD}=9.6$, $\mathrm{p}<.001)$. The QOL score decreased in all the life-space mobility categories over the follow-up period. The average decrease in the QOL score among those whose life-space had remained stable or improved over the two-year follow-up was -4.8 points $(\mathrm{SD}=9.3)$. In the other categories of change in life-space mobility the mean change in the QOL score was -7.6 points $(\mathrm{SD}=9.6$ ) for late decline, -7.2 points $(\mathrm{SD}=9.4)$ for early decline, and -7.6 points $(\mathrm{SD}=11.1$ ) for constant decline.

Compared to those whose life-space remained stable or improved, those with late (age and gender adjusted $\beta=4.13 \mathrm{p}=.004$, group*time $\mathrm{p}=.001)$ or early $(\beta=3.58, \mathrm{p}=.012$, group*time $\mathrm{p}=.006$ ) decline were more likely to show a decline in their QOL score. For those in the constant decline category, the group difference was borderline significant $(\beta=6.07 \mathrm{p}=.058)$, while the group*time interaction effect was no longer statistically significant ( $\mathrm{p}=.242)$. Adjusting for number of chronic conditions, cognitive impairments, lower extremity performance and years of education did not materially change these results (Table 3). 


\section{DISCUSSION}

This study shows that decline in life-space mobility is associated with decline in QOL among community-dwelling older people, even after taking into account several potential confounders. Overall, the QOL score decreased in all the life-space categories. Compared to those whose life-space remained stable or improved, the decline in QOL was greater among those whose life-space score decreased $>10$ points at any time during the follow-up. These findings suggest that irrespective of an older person's physical and cognitive functioning, maintenance of life-space mobility may help to maintain a good QOL

There may be several explanations for the association between the decline in life-space mobility and the decline in QOL. First, diminished possibilities for participation in outdoor activities and the resulting insufficient social interaction may lead to social isolation and loneliness, which, in turn, may lead to poor QOL $[12,31,32]$. Second, a reduction in time spent outdoors, and in consequence a lower level of physical activity and more sedentary behaviour, may have adverse effects on health and thus a negative influence on QOL [33]. Spending time outdoors may also have a direct effect on QOL, as people, especially in the Finnish context, often like to enjoy the outdoors, which has been shown to enhance their wellbeing [34-36]. Third, a decline in life-space mobility may indicate difficulties in taking care of daily errands independently, which may intensify feelings of losing control over one's life. Sense of autonomy is an important component of QOL in old age and also correlates with life-space mobility [16]. Finally, reduced life-space mobility may indicate losses in the social roles stemming from reduced opportunities for productive or other valued activities, such as helping others, contributing to civil society or enjoyment of art, all of which have health benefits [37]. 
In this study, the categorization of life-space mobility was based on a previously determined meaningful change of $>10$ points in the life-space mobility score $[14,24,28]$. Based on this categorization, the majority of the study participants maintained or improved their life-space mobility during the follow-up period. The proportion of participants whose life-space mobility declined throughout the follow-up was rather low; however, the average decline in this group was almost 37 points. A 37-point decline indicates very severe activity restriction and may lead to negative health outcomes. For example, a decline of 24 points has been found to increase mortality risk [20]. For QOL, to the authors' knowledge, no consensus exists on what can be considered as a meaningful change in the QOL score. It is arguable that any change in the WHOQOL scale has face value, as it reports "individuals' perceptions in the context of their culture and value systems, and their personal goals, standards and concerns" [1]. Consequently, especially among those whose life-space mobility declined during the follow-up, the observed decline in their QOL score was larger than it was among those who were able to maintain or improve their life-space mobility. This can be regarded as meaningful for the individual and hence we can make cautious assumptions on the causal association between life-space mobility and QOL.

Life-space mobility correlates with lower extremity performance [16], which is also a risk factor for poor QOL $[38,39]$. Thus, it seemed logical that lower extremity performance might explain the association between life-space mobility and QOL. However, adjusting the models for lower extremity performance did not change the results. The present result is in line with earlier research, where offering community-dwelling older people with severe mobility limitations opportunities for out-of-home activity improved the physical dimension of their 
QOL, even if it did not improve their lower extremity performance [40]. Further research should focus on interventions aiming at the maintenance of life-space mobility.

Among the strengths of the present study are the large population-based sample, two-year follow-up period and a focus on a topic which has not been widely studied previously. We were able to take into account changes in both the predictor and the outcome over time and investigate the changes in both. However, some limitations remain. It should be noted that over time, the meaning of the self-evaluation of QOL may change [41]. For example, as people adapt to their situation, decline in health and mobility may impact on their standards, goals and values [1], and thus aspects of life other than health or mobility may become more important, impacting in turn on their overall ratings of QOL. However, in this study we took of changes in life-space mobility over time, which reduces the risk of response shift as an explanation of the observed differences. However, we cannot rule out the possibility that response shift might have induced a slight underestimation of the strength of the association. In addition, QOL was assessed only at baseline and at the last follow-up, two years later, whereas life-space mobility was assessed annually. Consequently, we do not know precisely at what point during the follow-up the changes in QOL took place. Some of the baseline characteristics, such as number of chronic conditions or lower extremity performance, may have changed over time, and hence we cannot rule out the possibility that these changes underlie the association between change in life-space mobility and QOL. Moreover, other factors besides those that we considered may also underlie the association. However, we should bear in mind that QOL is multidimensional, and thus it is important to ensure that the other variables in the analyses do not conceptually overlap with the QOL dimensions. For example, we did not include depressive symptoms in the GEE models as covariates, as scales 
of depressive symptoms include items, such as sadness and anxiety, sleep quality and enjoyment of life that overlap with the psychological dimension of QOL.

In conclusion, the results of the present study highlight the importance of ensuring that older people have sufficient opportunities for out-of-home activities. Understanding the factors affecting out-of-home mobility and how best to promote it among older adults can help in finding ways for older people to stay engaged in the community, thereby supporting active ageing. 


\section{ACKNOWLEDGMENTS}

We thank all the study participants and interviewers for their contribution. We are grateful for Eeva-Maija Palonen, MSc, for her valuable work as coordinator of the LISPE project. The Gerontology Research Center (GEREC) is a joint effort between the Universities of Jyväskylä and Tampere, Finland.

\section{Funding sources}

This work was supported by the Academy of Finland (the Future of Living and Housing Program ASU-LIVE; grant number 255403 to [TR]), and number 263729 to [AV]; Finnish Ministry of Education and Culture to [TR] and [EP]; Ribbingska Foundation in Lund, Sweden to [SI].

\section{Author Contributions}

The authors are justifiably credited with authorship, according to the authorship criteria. MR: conception, design, data collection, statistical analysis and interpretation of the data, drafting the article; EP: conception, design, data collection, critical revision of the article; AV: conception, design, data collection, critical revision of the article. SI: conception, design, critical revision of the article; MK: Statistical analysis and interpretation of the data, TR: conception, design, data collection, critical revision of the article, PI for the LISPE project. All the authors approved the final manuscript.

\section{Compliance with Ethical Standards}

Ethical approval: All procedures performed in studies involving human participants were in accordance with the ethical standards of the institutional and/or national research committee and with the 1964 Helsinki Declaration and its later amendments or comparable ethical 
standards. The LISPE project was approved by the Ethical Committee of the University of Jyväskylä, Finland.

Informed consent: Informed consent was obtained from all individual participants included in the study.

Conflict of Interest: The authors declare no conflicts of interest. 


\section{REFERENCES}

1. The WHOQOL Group. (1998). Development of the World Health Organization WHOQOL-BREF quality of life assessment. Psychological Medicine, 28, 551-558.

2. Netuveli, G., \& Blane, D. (2008). Quality of life in older ages. British Medical Bulletin, $85,113-126$.

3. Netuveli, G., Wiggins, R. D., Hildon, Z., Montgomery, S. M., \& Blane, D. (2006). Quality of life at older ages: evidence from the English longitudinal study of aging (wave 1). Journal of Epidemiology and Community Health, 60(4), 357-363.

4. Blane, D., Netuveli, G., \& Montgomery, S. M. (2008). Quality of life, health and physiological status and change at older ages. Social Science \& Medicine (1982), 66(7), 1579-1587.

5. Wilhelmson, K., Andersson, C., Waern, M., \& Allebeck, P. (2005). Elderly people's perspectives on quality of life. Ageing \& Society, 25(4), 585-600.

6. World Health Organization. (2002). ICF: International Classification of Functioning, Disability and Health. Geneva: World Health Organization.

7. World Health Organization (WHO). (2002). Active Ageing: A policy framework.

8. Theis, K. A., Murphy, L., Hootman, J. M., \& Wilkie, R. (2013). Social participation restriction among US adults with arthritis: a population-based study using the International Classification of Functioning, Disability and Health. Arthritis Care \& Research, 65(7), 10591069.

9. Wilkie, R., Peat, G., Thomas, E., \& Croft, P. (2006). The prevalence of person-perceived participation restriction in community-dwelling older adults. Quality of Life Research, 15(9), 1471-1479.

10. Olsson, A., Lampic, C., Skovdahl, K., \& Engstrom, M. (2013). Persons with early-stage dementia reflect on being outdoors: a repeated interview study. Aging \& Mental Health, 17(7), 793-800.

11. Rejeski, W. J., \& Mihalko, S. L. (2001). Physical activity and quality of life in older adults. The journals of gerontology.Series A, Biological Sciences and Medical Sciences, 56 Spec No 2, 23-35.

12. Ekstrom, H., Ivanoff, S. D., \& Elmstahl, S. (2008). Restriction in social participation and lower life satisfaction among fractured in pain: results from the population study "Good Aging in Skane". Archives of Gerontology and Geriatrics, 46(3), 409-424.

13. Levasseur, M., Desrosiers, J., \& St-Cyr Tribble, D. (2008). Do quality of life, participation and environment of older adults differ according to level of activity? Health and Quality of Life Outcomes, 6, 30. 
14. Baker, P. S., Bodner, E. V., \& Allman, R. M. (2003). Measuring life-space mobility in community-dwelling older adults. Journal of the American Geriatrics Society, 51(11), 16101614.

15. Sawyer, P., \& Allman, R. M. (2010). Resilience in mobility in the context of chronic disease and aging:cross-sectional and prospective findings from the University of Alabama at Birmingham (UAB) Study of Aging. In P. S. Fry, \& C. L. M. Keyes (Eds.), Frontiers of resilient aging:Life-strengths and wellness in late life. (pp. 310-339). New York: Cambridge University Press.

16. Portegijs, E., Rantakokko, M., Mikkola, T. M., Viljanen, A., \& Rantanen, T. (2014). Association Between Physical Performance and Sense of Autonomy in Outdoor Activities and Life-Space Mobility in Community-Dwelling Older People. Journal of the American Geriatrics Society, 62(4), 615-621.

17. Lo, A. X., Brown, C. J., Sawyer, P., Kennedy, R. E., \& Allman, R. M. (2014). Life-space mobility declines associated with incident falls and fractures. Journal of the American Geriatrics Society, 62(5), 919-923.

18. Xue, Q. L., Fried, L. P., Glass, T. A., Laffan, A., \& Chaves, P. H. (2008). Life-space constriction, development of frailty, and the competing risk of mortality: the Women's Health And Aging Study I. American Journal of Epidemiology, 167(2), 240-248.

19. Sheppard, K. D., Sawyer, P., Ritchie, C. S., Allman, R. M., \& Brown, C. J. (2013). Lifespace mobility predicts nursing home admission over 6 years. Journal of Aging and Health, 25(6), 907-920.

20. Mackey, D. C., Cauley, J. A., Barrett-Connor, E., Schousboe, J. T., Cawthon, P. M., Cummings, S. R., et al. (2014). Life-space mobility and mortality in older men: a prospective cohort study. Journal of the American Geriatrics Society, 62(7), 1288-1296.

21. Boyle, P. A., Buchman, A. S., Barnes, L. L., James, B. D., \& Bennett, D. A. (2010). Association between life space and risk of mortality in advanced age. Journal of the American Geriatrics Society, 58(10), 1925-1930.

22. Rantakokko, M., Portegijs, E., Viljanen, A., Iwarsson, S., \& Rantanen, T. (2013). LifeSpace Mobility and Quality of Life among Community-Dwelling Older People. Journal of the American Geriatrics Society, 61, 1830-1832.

23. Rantanen, T., Portegijs, E., Viljanen, A., Eronen, J., Saajanaho, M., Tsai, L. T., et al. (2012). Individual and environmental factors underlying life space of older people - study protocol and design of a cohort study on life-space mobility in old age (LISPE). BMC Public Health, 12, 1018-2458-12-1018.

24. Portegijs, E., Iwarsson, S., Rantakokko, M., Viljanen, A., \& Rantanen, T. (2014). Lifespace mobility assessment in older people in Finland; measurement properties in winter and spring. BMC Research Notes, 7(1), 323-0500-7-323.

25. Galobardes, B., Lynch, J., \& Smith, G. D. (2007). Measuring socioeconomic position in health research. British Medical Bulletin, 81-82, 21-37. 
26. Folstein, M. F., Folstein, S. E., \& McHugh, P. R. (1975). "Mini-mental state". A practical method for grading the cognitive state of patients for the clinician. Journal of Psychiatric Research, 12(3), 189-198.

27. Guralnik, J. M., Simonsick, E. M., Ferrucci, L., Glynn, R. J., Berkman, L. F., Blazer, D. G., et al. (1994). A short physical performance battery assessing lower extremity function: association with self-reported disability and prediction of mortality and nursing home admission. Journal of Gerontology, 49(2), M85-94.

28. Kammerlind, A. S., Fristedt, S., Ernsth Bravell, M., \& Fransson, E. I. (2014). Test-retest reliability of the Swedish version of the Life-Space Assessment Questionnaire among community-dwelling older adults. Clinical Rehabilitation, 28(8), 817-823.

29. Liang, K. Y., \& Zeger, S. L. (1986). Longitudinal data analysis using generalized linear models. Biometrika, 13-22.

30. Azur, M. J., Stuart, E. A., Frangakis, C., \& Leaf, P. J. (2011). Multiple imputation by chained equations: what is it and how does it work? International Journal of Methods in Psychiatric Research, 20(1), 40-49.

31. Gilmour, H. (2012). Social participation and the health and well-being of Canadian seniors. Health reports / Statistics Canada, Canadian Centre for Health Information 23(4), 2332.

32. Ekwall, A. K., Sivberg, B., \& Hallberg, I. R. (2005). Loneliness as a predictor of quality of life among older caregivers. Journal of Advanced Nursing, 49(1), 23-32.

33. Balboa-Castillo, T., Leon-Munoz, L. M., Graciani, A., Rodriguez-Artalejo, F., \& GuallarCastillon, P. (2011). Longitudinal association of physical activity and sedentary behavior during leisure time with health-related quality of life in community-dwelling older adults. Health and Quality of Life Outcomes, 9, 47-7525-9-47.

34. Korpela, K. M., Ylen, M., Tyrvainen, L., \& Silvennoinen, H. (2010). Favorite green, waterside and urban environments, restorative experiences and perceived health in Finland. Health Promotion International, 25(2), 200-209.

35. Pasanen, T. P., Tyrvainen, L., \& Korpela, K. M. (2014). The Relationship between Perceived Health and Physical Activity Indoors, Outdoors in Built Environments, and Outdoors in Nature. Applied Psychology, Health and Well-being, 6(3), 324-346.

36. Bowler, D. E., Buyung-Ali, L. M., Knight, T. M., \& Pullin, A. S. (2010). A systematic review of evidence for the added benefits to health of exposure to natural environments. BMC Public Health, 10, 456-2458-10-456.

37. Pynnonen, K., Tormakangas, T., Heikkinen, R. L., Rantanen, T., \& Lyyra, T. M. (2012). Does social activity decrease risk for institutionalization and mortality in older people? The Journals of Gerontology. Series B, Psychological Sciences and Social Sciences, 67(6), 765774. 
38. Alexandre Tda, S., Cordeiro, R. C., \& Ramos, L. R. (2009). Factors associated to quality of life in active elderly. Revista de Saude Publica, 43(4), 613-621.

39. Karakaya, M. G., Bilgin, S. C., Ekici, G., Kose, N., \& Otman, A. S. (2009). Functional mobility, depressive symptoms, level of independence, and quality of life of the elderly living at home and in the nursing home. Journal of the American Medical Directors Association, 10(9), 662-666.

40. Rantanen, T., Ayravainen, I., Eronen, J., Lyyra, T., Tormakangas, T., Vaarama, M., et al. (2015). The effect of an outdoor activities' intervention delivered by older volunteers on the quality of life of older people with severe mobility limitations: a randomized controlled trial. Aging Clinical and Experimental Research, 27(2), 161-169.

41. Schwartz, C. E., \& Sprangers, M. A. G. (1999). Methodological approaches for assessing response shift in longitudinal health-related quality-of-life research. Social Science \& Medicine, 48(11), 1531-1548. 
Table 1. Life-space mobility score in categories based on changes in life-space mobility score over the two-year follow-up period $(n=751)$.

\begin{tabular}{|c|c|c|c|c|c|c|c|c|c|c|c|}
\hline \multirow{3}{*}{$\begin{array}{l}\text { Life-space } \\
\text { mobility } \\
\text { category }\end{array}$} & \multirow[b]{3}{*}{$\mathrm{n}$} & \multirow{2}{*}{\multicolumn{2}{|c|}{ Baseline }} & \multirow{2}{*}{\multicolumn{2}{|c|}{$\begin{array}{c}1-y e a r \\
\text { follow-up }\end{array}$}} & \multirow{2}{*}{\multicolumn{2}{|c|}{$\begin{array}{l}\text { 2-year } \\
\text { follow-up }\end{array}$}} & \multicolumn{4}{|c|}{ 2-year change } \\
\hline & & & & & & & & \multicolumn{2}{|c|}{ score } & \multicolumn{2}{|c|}{$\%$} \\
\hline & & Mean & SD & Mean & SD & Mean & SD & Mean & SD & & s.e. \\
\hline Maintained & 335 & 58.9 & 19.6 & 63.6 & 20.4 & 66.0 & 22.1 & +7.1 & 11.7 & +14.9 & 1.7 \\
\hline Late decline & 178 & 64.4 & 17.5 & 76.0 & 18.6 & 55.2 & 19.1 & -9.3 & 14.0 & -13.6 & 1.9 \\
\hline Early decline & 211 & 73.5 & 19.3 & 53.4 & 20.3 & 61.5 & 22.0 & -12.0 & 12.5 & -17.3 & 1.3 \\
\hline $\begin{array}{l}\text { Constant } \\
\text { Decline }\end{array}$ & 27 & 83.9 & 19.8 & 64.0 & 21.9 & 42.3 & 22.9 & -36.9 & 12.8 & -46.6 & 1.3 \\
\hline
\end{tabular}

Maintained: Life-space stable over the follow-up

Late decline: Life-space stable at one-year but declined at two-year follow-up

Early decline: Life-space declined at one-year but stable at two year follow-up

Constant decline: Life-space declined at one- and two-year follow-ups. 
Table 2. Baseline characteristics of the 75- to 90-year-old participants of the life-space mobility in old age (LISPE) project, categorized according to changes in life-space mobility $(\mathrm{n}=751)$.

\begin{tabular}{lccccc}
\hline & \multicolumn{4}{c}{ Categorization based on changes in life-space mobility } & \\
\cline { 2 - 5 } & Maintained & Late decline & Early decline & $\begin{array}{c}\text { Constant } \\
\text { decline } \\
\mathrm{n}=27\end{array}$ & \\
& $\mathrm{n}=335$ & $\mathrm{n}=178$ & $\mathrm{n}=211$ & 59.3 & .587 \\
\hline Women, \% & 65.7 & 62.4 & 60.2 & & \\
& & & & & \\
& Mean $\pm \mathrm{SD}$ & Mean $\pm \mathrm{SD}$ & Mean $\pm \mathrm{SD}$ & Mean $\pm \mathrm{SD}$ & \\
Age, years & $80.4 \pm 4.1$ & $80.3 \pm 4.1$ & $80.6 \pm 4.3$ & $79.9 \pm 3.7$ & .862 \\
Education, years & $9.6 \pm 3.9$ & $9.4 \pm 3.8$ & $10.0 \pm 4.8$ & $10.6 \pm 4.7$ & .343 \\
MMSE, score & $26.5 \pm 2.6$ & $25.8 \pm 2.6$ & $26.6 \pm 2.6$ & $26.1 \pm 3.0$ & .023 \\
$\begin{array}{l}\text { SPPB, score } \\
\text { Chronic }\end{array}$ & $9.7 \pm 2.5$ & $9.8 \pm 2.1$ & $9.9 \pm 2.3$ & $9.9 \pm 1.8$ & .870 \\
conditions, & $4.3 \pm 2.5$ & $4.5 \pm 2.6$ & $4.3 \pm 2.3$ & $4.3 \pm 1.7$ & .872 \\
number & & & & & \\
\hline
\end{tabular}

MMSE, Mini-Mental State Examination

SPPB, Short Physical Performance Battery 
Table 3. Change in the QOL score over the follow-up according to the life-space mobility categories at two-year follow-up ( $\mathrm{n}=751)$.

\begin{tabular}{|c|c|c|c|c|c|c|}
\hline \multirow[b]{3}{*}{$\begin{array}{l}\text { Life-space } \\
\text { mobility } \\
\text { category }\end{array}$} & \multicolumn{2}{|c|}{ QOL score } & & & \multirow[b]{3}{*}{$\begin{array}{l}\text { Group } \\
\text { difference } \\
\text { p-valueł }\end{array}$} & \multirow[b]{3}{*}{$\begin{array}{l}\text { Group*time } \\
\text { interaction } \\
\text { p-value }\end{array}$} \\
\hline & Baseline & Follow-up & & & & \\
\hline & Mean \pm SD & Mean \pm SD & $\beta$ & s.e. & & \\
\hline Maintained & $100.6 \pm 12.2$ & $95.8 \pm 14.0$ & \multicolumn{2}{|c|}{ ref. } & ref. & ref. \\
\hline Early decline & $102.0 \pm 15.5$ & $94.8 \pm 12.7$ & 3.41 & 1.38 & .013 & .005 \\
\hline Late decline & $101.7 \pm 11.7$ & $94.0 \pm 14.1$ & 4.76 & 1.42 & .001 & .001 \\
\hline Constant decline & $104.8 \pm 10.0$ & $97.1 \pm 15.5$ & 5.69 & 2.98 & .056 & .261 \\
\hline
\end{tabular}

₹ GEE analyses.

Group*time interaction for time-related change in QOL score in the life-space mobility categories, adjusted for age, gender, education, number of chronic conditions, lower extremity performance and cognitive functioning.

Maintained: Life-space stable over the follow-up

Late decline: Life-space stable at one-year but declined at two-year follow-up

Early decline: Life-space declined at one-year but stable at two year follow-up

Constant decline: Life-space declined at one- and two-year follow-ups. 


\section{FIGURE LEGENDS}

Figure 1. Study flow of the Life-space mobility in old age (LISPE) project 2012-2014. 
Figure 1.

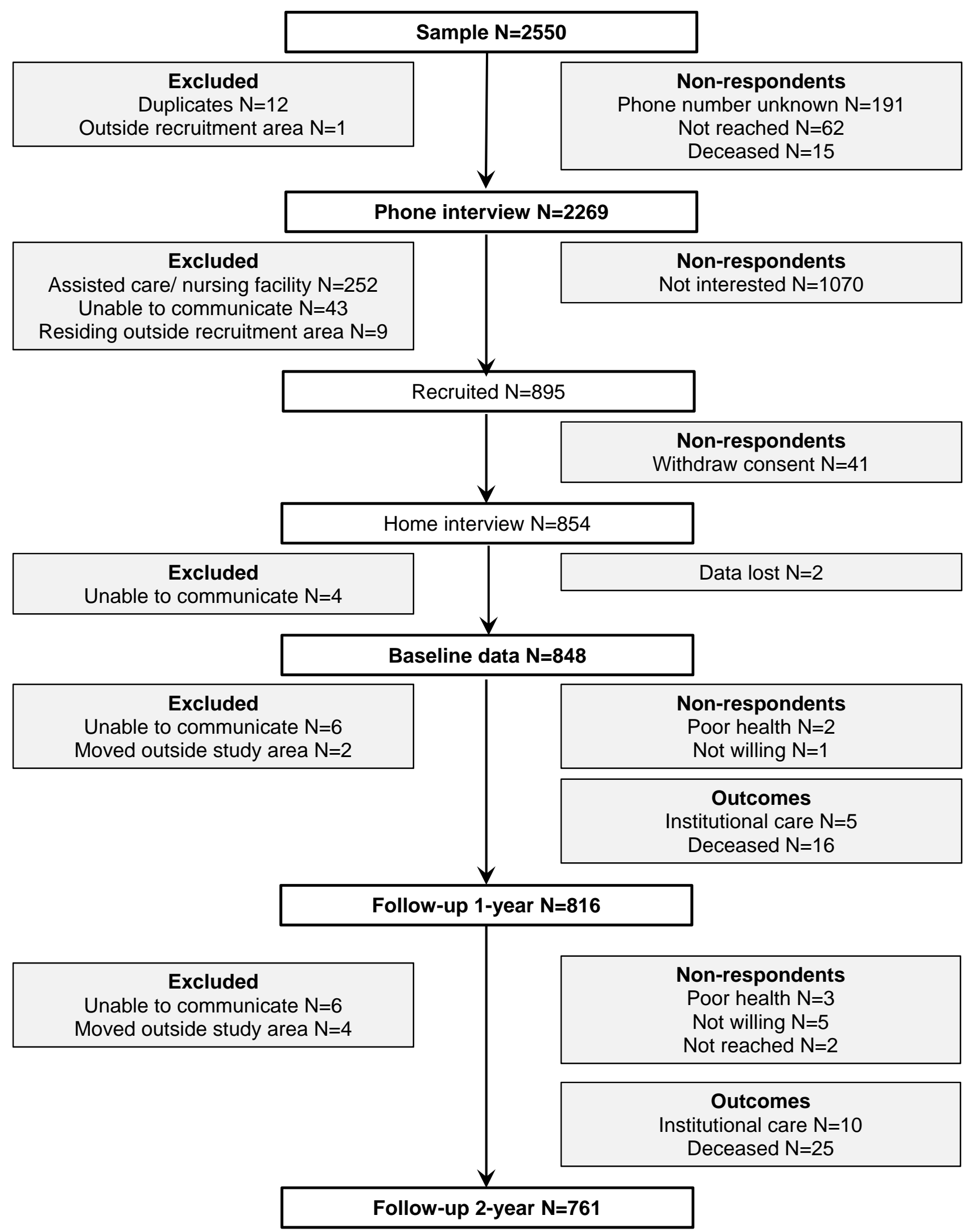


